XIX.

\title{
Untersuchungen an Verbrechern über die morphologischen Veränderungen der 0hrmuschel.
}

\author{
Yon \\ Dr. Erust Váli \\ in Bndapest.
}

(Von der Redaction ibernommen am 27. September 1892.)

Es ist eine längst verbreitete und täglich mehr Raum gewinnende Ansicht der Psychiatrie, dass bei belasteten Geisteskranken nicht blos die Symptome der Geistesstörung degeneriren, sondern auch die einzelnen Körpertheile untrïgliche Anzeichen der Degeneration aufweisen.

Unter den Psychiatern war Morel der erste, der bei Geisteskranken die äusseren anatomischen Veränderungen mit den Sym. ptomen der Geistesstörung zugleich beobachtete, und gehen diese nach seiner Ansicht derart Hand in Hand, dass bei Feststellung der Diagnose auch die anatomische Veränderung einen Stützpunkt bildet. So wird z. B. von den anatomischen Veränderungen der Gestaltang des Schädels, Asymmetrie der beiden Gesichtshälften, der abnormen Entwicklung und Anordnung der Zähne, der Missbildung der Zunge, Uvula, Gaumen und des Ohres, der Verkrïmmung der Sexualorgane u. s. w. u. s. w. Seitens der Psychiater heutzutage ebenso viel Aufmerksamkeit geschenkt, wie den f u $\mathrm{nc}$ tionellen Störungen, z. B. Muskelzuckungen, Sprachstörungen, Taubstummbeit, Schielen, Nystagmus, Impotenz, sexueller Erregtheit, Neuralgien u. s. w. u. s. w.

Bei Geisteskranken, Idioten und Verbrechern finden wir die meisten anatomischen Veränderungen an der Ohrmuschel; die Psychiater selbst fassten die an der Obrmuschel ibrer Kranken beobachteten anatomischen Veränderungen unter eine Collectiv- 
benennung zusammen, indem sie die derart veränderte Ohrmuschel Morel'sches Ohr benannten.

Unsererseits können wir uns mit dieser allgemeinen Bezeichnung nicht zufrieden geben. Die morphologischen Veränderungen der einzelnen anatomischen Theile der Obrmuschel sind bereits ins Reine gebracht; dieser Gegenstand bedarf nur noch nach der eimen Richtung der Aufklärung, in welchem Zahlenverhältnisse diese Veränderungen bei gesunden und freien, also normalen Individuen, Geisteskranken, Idioten und Verbrechern vorkommen, mit anderen Worten, ob die Anomalien der Ohrmuschel als degeneratives Symptom betrachtet werden diurfen oder nicht.

In erster Reihe waren die Phrenologen und Pbysiognomen bestrebt, aus den morphologischen Eigenthumlichkeiten der einzelnen Theile des Gesichtes und des Scbädels auf die Intelligenz, den Charakter des Individuums Schlüsse zu ziehen. Eine Physiognomie, die im Jahre 1860 in italienischer Sprache erschien, gelangt zu folgendem Resultat: „Kleine, schön geformte Ohrmuscheln sind Zeichen hoher geistiger Entwicklung; hingegen deuten grosse Ohrmuscheln anf ein langes Leben ihres Besitzers, jedoch auf schwächeren Verstand. Zu geistiger Arbeit Geneigte haben schön gestaltete, kleine Ohrmuscheln, während die mit grossen und dicken Museheln Versehenen zumeist undisciplinirt sind. Menschen mit länglichen und spitzen Ohrmuscheln sind meistentheils hinterlistig."

Nach diesen Laien der Wissenschaft erheben die Anthropologen ihr pro und contra über die Degeneration der Ohrmuschel; bald erscheinen die Psychiater anf dem Plane, die neben den übrigen Anomalien des Körpers denjenigen der Ohrmuschel besondere Aufmerksamkeit widmen. Binder spricht zuerst die Ansicht ans, dass bei Geisteskranken und Idioten kein Organ so sehr degenerirt, wie gerade die Obrmuschel.

Obwohl dieser Gegenstand vielmehr in das Gebiet der Psychiatrie gehört, so ist es nach meinem Dafürhalten doch unsere Pflicht, auf Grund exacter Untersucbungen und deren Ergebnisse zur Lösung der Streitfrage beizutragen, da wir als Obrenärzte uns berufsmässig mit den Ohrenkrankbeiten befassen und mithin in den Stand gesetzt sind, die Anomalien der Musehel auf Grund zahlloser Fälle zu beurtheileo.

In den vor zwei Jahren erschienenen Heften des Archivs für Ohrenheilkunde publicirte der italienische Obrenarzt $\mathrm{Gradenigo}$ seine diesbezüglichen zahlreichen Untersuchungen. Meines Wissens 
war er der erste Ohrenarzt, der auf Grund genauer und zahlreicher Messungen an normalen Individuen, Geisteskranken, Idioten und Verbrechern sich in die bisher theoretische Discussion mengte.

Die Angaben Gradeni go's zu controliren, war meine Absicht, als ich vor einem Jahre in Budapest ebenfalls Untersuchungen auf ähnlicher Basis anstellte. Ich untersuchte damals von normalen Individuen 500 Männer und 500 Frauen (zusammen 1000), 216 männliche und 188 weibliche Geisteskranke (zusammen 404), des Weiteren 59 männliche und 32 weibliche Idioten (zusammen 90). Die Ergebnisse dieser Untersuchungen warden im Jahrgange 1891 des Orvosi Hetilap publicirt.

Erst in diesem Jahre kam ich in die glitckliche Lage, auch an Verbrechern Untersuchungen anstellen zu können, und konnte ich mithin die Angaben Gradenigo's auch nach dieser Richtung: controliren. $\mathrm{Zn}$ diesem Behufe untersuchte ich in den Zuchtbäusern von Waitzen und Maria Nostra 500 männliche und 324 weibliche Verbrecher ${ }^{1}$ ) (zusammen 824). Ueber die hier gefundenen Daten will ich nun Rechenschaft abgeben, wobei ich bemerke, dass die bereits publicirten Angaben blos des Vergleichs halber stellenweise erwähnt werden sollen.

Von den zahlreichen Anomalien der Ohrmuschel zog ich blos die folgenden, in der Praxis häufiger vorkommenden Formen in Betracht:

Abstehende und asymmetrische Muscheln. Einfach angewachsenes $\mathrm{Ohrläppchen.} \mathrm{Längliches} \mathrm{und} \mathrm{zum} \mathrm{Gesicht} \mathrm{unter} \mathrm{einem}$ spitzen Winkel angewachsenes Läppchen. Grosses und dickes Läppchen. Den Helix uiberragender Anthelix. Mangel haft entwickeltes Crus sup. antehelicis. Bandförmiger Helix. Kurze, fehlende Fossa scaphoidea, verlängert oder überbriickt. MacacusOhr (der Helix ist mit zugespitztem Ende ausgedehnt). Helix und Anthelix gänzlich oder theilweise verwachsen; endlich die Darwin'schen Höcker.

I. Abstehende Muscheln. Unter normalen Verhältnissen macht die Muschel mit der lateralen Schädelfläche einen spitzen Winkel, d. h. der Angulus auriculo-temporalis ist ein spitzer Winkel. Ist dieser Winkel ein rechter, so entstehen die a b-

1) An dieser Stelle sage ich meinen verbindlichsten Dank den Leitern obiger Anstalten, der hochwärdigen Schwester Dominica Schreiner und den Herren Stefan Balkay und Benj. Dávid, die mich bei meinen Untersuchungen mit herzlicher Zuvorkommenheit unterstützten. 
stehenden Obren. Das Abstehen der Muscheln vom Ohre beruht auf' embryologischer Basis und ist die typischeste unter sämmtlichen Anomalien. Sie ist auch noch deshalb auffallend, weil sich ihr andere Anomalien zuzugesellen pflegen, wie da sind: dickes und bäufig angewachsenes, grosses Läppehen, mangelhafte Entwicklung des Crus sup. anthelicis; letzteres fehlt auch zuweilen gänzlich.

Beiderseitig abstehende Muscheln fand ich unter

$\begin{array}{cccc} & \text { Männern } & & \\ \text { bei normalen Individuen } & \text { Geisteskranken } & \text { Idioten } & \text { Verbrechern } \\ 16,8 & 36,5 & 34,4 & 18,6 \text { Proc. } \\ & \text { Weibern } & & \\ 10,5 & 23,8 & 25 & 15,4 \text { Proe. }\end{array}$

Blos linksseitig abstehende Muscheln fand ich insgesammt bei 6 Verbrechern, was 1,2 Proc. entspricht. Aus diesen Daten ersehen wir, dass, während ich bei Geisteskranken und Idioten mebr als doppelt so viel abstehende Muscheln fand, wie bei normalen Individuen, dieselbe Anomalie bei männlichen Verbrechern nur um 2 Proc. mehr besteht, bei weiblichen bingegen um mehr als die Hälfte. Gradenigo fand bei Verbrechern einen viel höheren Procentsatz.

Die Dimensionen der Muscheln. In Bezug auf Grössedimensionen unterscheiden wir viererlei Muscheln, und zwar ovale, in der Longitudinalrichtung verlängerte, transversal vergrösserte und dreieckige Muscheln, bei welch letzteren die obere Partie der Muschel viel breiter ist, als die untere.

Gelegentlich meiner Untersuchungen an Verbrechern habe ich, durch Herrn Prof. Aurel Török freundlichst aufmerksam gemacht, die Grösse der Muschel mittelst Schieberzirkel genan bestimmt. Die detaillirte Aufarbeitung der auf diese Art gefundenen Daten mir für eine andere Gelegenheit vorbehaltend, will ich hier nur im Allgemeinen andeuten, dass ich betreffs der Dimension die mannigfaltigsten Ohrmuscheln gefunden habe. So fand ich beiderseitig vollkommen gleiche Muscheln bei 8,4 Proe. der männlichen, 14,2 Proc. der weiblichen Verbrecher. Bei älteren Verbrechern fand ich häufig die auffallende Asymmetrie, dass z. B. die Breite einer $70 \mathrm{Mm}$. langen Muschel blos 22-24 Mm. betrug! Eine derartige unverhältnissmässige Vergrösserung des Querdurchmessers fand ich ebenfalls, doch viel seltener. Dreieckige Muscheln waren bei männlichen und weiblichen Verbrechern mit 8 Proc. vertreten. 
II. Das Obrläppchen entwickelt sich unter den Theilen der Muschel am spätesten; als secundäres Gebilde hat es geringere morphologische Bedeutung. Von seinen zahlreichen Anomalien zog ich nur folgende in Betracht: 1) Uebermässige Entwicklung, 2) Mangel des Läppchens, 3) behaartes Läppchen, 4) einfach und spitz angewachsenes Läppchen.

Ich stellte fest

bei Männern

Normale Geistes- Idioten Verbrecher kranke

(grosses, dickes Läppchen. . $6,9 \quad 12,5 \quad 18,9 \quad 9,4$ Proc.

behaartes Läppchen . . . $2,0 \quad 4,0 \quad 1,7 \quad 8,2=$

mangelhaft entwickeltes

Läppchen . . . . . . - - $0,9 \quad 1,7 \quad 1,4=$

einfach angewachsenes

Läppchen . . . . . . $9,2 \quad 9,2 \quad 15,3 \quad 6,4=$

spitz angewachsenes Läppchen $\quad 5,6 \quad 13,4 \quad 12,4 \quad 8,8=$

bei Weibern

grosses, dickes Lüppchen . . $\begin{array}{llll}2,0 & 10,9 & 25,0 & 7,0 \\ \end{array}$

behaartes Läppohen

mangelhaft entwickeltes

Läppchen

einfach angewachsenes

Läppchen . . . . . . $\begin{array}{ccccc}9,2 & 7,7 & 12,4 & 6,3 & =\end{array}$

spitz angewachsenes Läppchen $\quad 8,8 \quad 8,4 \quad 9,8 \quad 8,8=$

Aus dieser Tafel ist klar ersichtlich, dass an dem Läppehen der Geisteskranken und Idioten zahlreiche Anomalien zu finden sind; meine diesbezüglichen Daten sind mit jenen Gradenigo's identisch, doch ist der gefundene Procentsatz bei Verbrechern bedeutend kleiner, als Gradenigo angiebt, überragt jedoch denjenigen bei Normalen auch hier ganz bedentend.

Das spitz angewachsene Läppchen hält Gradenigo für ein sehr wichtiges degeneratives Symptom, und er fand thatsächlich bei Verbrechern einen hoben Procentsatz. Meinerseits konnte ich einen solchen nur bei männlichen Verbrechern aufweisen, bei weiblichen fand ich diese Anomalie in gleicher Anzahl wie bei den ubrigen untersuchten Weibern.

Eine sehr seltene Erscheinung bildet es und verleiht dem Gesichte einen eigenthïmlichen Ausdruck, wenn die soeben erwähnten Anomalien nur eins eitig vorkommen. Unter den männlichen Verbrechern fanden sich sämmtliche Anomalien in 2-3 Exemplaren, bei weiblicben jedoch sah ich blos in zwei Fallen, dass das Läppchen auf das Gesicht spitz angewachsen war. Die 
äbrigen Anomalien waren bei weiblichen Verbrechern alle beiderseitig.

Gestiitzt auf die gefundenen Daten, bin ich geneigt, das grosse und beharte Läppchen als ein degeneratives Symptom zu betrachten. Bei Frauen ist diese Anomalie ausserordentlich selten; bei normalen Männern ebenfalls, hingegen finden wir sie bei Geisteskranken und Verbrechern in beträchtlicher Anzahl.

III. Der Helix erleidet embryologiseh die grössten Modificationen, weshalb an demselben die degenerativen Symptome nicht selten sind. Am häufigsten ragt er bis zur selben Höhe, wie der Anthelix, bald zieht er sich bis zur Mitte des Läppchens herab; in anderen Fällen wiederum endet er schon dort, wo er ans der Querrichtung in die absteigende Portion übergehen soll.

Diese Anomalien werden durch folgende Beobachtungen bekräftigt:

Beiderseitig mangelhaft entwickelten Helix fand ich bei normalen Männern 3,2 Proc., Geisteskranken 9,7 Proc., Idioten 8,1 Proc. und Verbrechern 6,4 Proc.; unter den Weibern hingegen bei Normalen 6,2, Geisteskranken 6,5, Idioten 9,1 und Verbrecherinnen 9,8 Proc. Blos rechtsseitig mangelhaft entwickelten Helix bei Verbrechern 1,2 Proc., lin $\mathrm{k}$ s s e itige n 0,6 Proc.; bei Verbrecherinnen dieselben Anomalien nur in je 2 Faillen.

Beiderseitigen gänzlichen Mangel des Helix fand ich blos bei männlichen Idioten 0,8 Proc. und Verbrechern 0,4 Proc.; bei weiblichen Idioten und Verbrecherinnen 0,6 Proc., bei Geisteskranken 0,5 Proc. Blos rechtsseitig fehlenden Helix fand ich bei Verbrechern 6,4 Proc., Verbrecherinnen 0,6 Proc.

Wie die bisherigen Ziffernangaben also zeigen, weisen diese Anomalien bei Geisteskranken, Idioten und Verbrechern einen viel höheren Procentsatz auf, als bei normalen Individuen; besonders zahlreich aber sind die Weiber von diesen Anomalien betroffen.

Beiderseitigen bandförmigen Helix fand ich bei normalen Individuen 4,4, Geisteskranken 6,4, Idioten 12,4 and Verbrechern 5,8 Proc.; unter den Weibern bei Normalen 3,6, Geisteskranken 7,5, Idioten 12,5 und Verbrecherinnen 8,1 Proc. Nur rechtsseitigen bandförmigen Helix bei Verbrechern und Verbrecherinnen je 1,2 Proc, nur linksseitigen bei Verbrechern 0,8 Proc. Gradenigo sah diese Anomalien bei Verbrecherinnen bedeutend seltener. 
Den beiden Formen des Darwin'schen Ohres, dem Macacusohr und den Darwin'schen Höckern, kommt insofern Bedeutung zu, als anf Grund der vergleichenden Anatomie wahrscheinlich ist, dass diese beiden Deformitäten eine constante Eigenheit der ausgegradeten Ohrmuschel der Säugethiere bilden. Ich fand bei

\begin{tabular}{|c|c|c|c|c|c|c|}
\hline \multicolumn{7}{|c|}{ Männern } \\
\hline & & an & Normalen & Geisteskranken & Idioten & Verbrechern \\
\hline Darwin'sche & Höcker & . & . 2,4 & 4,1 & 3,4 & 1,2 Proc. \\
\hline Macacusohr & 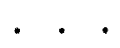 & & 0,6 & 2,3 & 3,4 & $2,1=$ \\
\hline \multicolumn{7}{|c|}{ Weibern } \\
\hline ar & Höcker & . & . 4,0 & 3,6 & 3,2 & - Proc. \\
\hline Macacusohr & 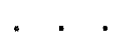 & . & 0,4 & 3,5 & - & - \\
\hline
\end{tabular}

Darwin'sche Höcker fand ich nur links bei Verbrechern 1,8, Verbrecherinnen 1,5 Proc.; Macacusobr nur links bei Verbrechern 0,8 Proc. Idioten und Geisteskranke haben den höchsten Procentsatz; bei Verbrechern beiderlei Geschlechts wiederum finden sich am linken Ohre die meisten Deformitäten.

Eine äusserst seltene Anomalie des Helix konnte ich wiederholt bei Verbrechern constatiren, eine Leiste nämlich, die sich vom Helix aus gegen den Anthelix fortsetzt (Proc. crur. helic. ad antehel.). Beim Menschen ist dies ein embryologisehes Residuum, bei höher gearteten Thieren hingegen finden wir es wohl entwickelt.

IV. Die Deformitaten des Anthelix. Eine eigenthümliche Form nimmt das Ohr dann an, wenn der Anthelix den Helix iuberragt. Diese Form wird Wildermuth'sches Ohr I genannt und kommt hänfiger besonders bei Weibern vor. So fand ich beiderseitiges Wildermuth'sches Ohr bei normalen Individuen 5,6, Geisteskranken 8,8, Idioten 12,4, Verbrechern 9,2 Proc.; unter Weibern bei Normalen 6,0, Geisteskranken 8,4, Idioten 24,3, Verbrecherinnen 16,9 Proc. Blos rechtsseitig bei Verbrechern 2,0, nur linksseitig 1,1 Proc. Bei Verbrecherinnen sah ich einseitige Formen nur in einigen Fällen.

Dreitheilung des Anthelix ist eine sehr seltene Erscheinung; in solchen Fällen ist der dritte Schenkel nur rudimentär entwickelt. Bei normalen Individuen und Geisteskranken finden wir diese Deformität kaum, bei männlichen Idioten fand ich eine solche beiderseitige Form 1,4, bei Verbrechern 0,8, Verbrecherinnen 1,5 Proc; nur reehtsseitig bei Verbrechern 1,0 Proc. Dass diese Form in Gesellschaft mit Macacusohr auftrete, wie Gradenigo fand, habe ich nicht beobachtet. 
Das Crus superius kann entweder vollkommen oder partiell entwickelt sein. Von 500 untersuchten Verbrechern fand ich es ein einziges Mal gänzlich fehlend, beiderseitig mangelhaft entwickelt bei Verbrechern nur 0,8 Proc, hingegen steigt seine Zahl bei Geisteskranken und Idioten auch anf 5-6 Proc. Bei Verbrecherinnen fand ich auf diesem Gebiete keine Abnormität.

Der Helix verwächst mit dem Anthelix gewöhnlich nur partiell; eine vollkommene Verwachsung ist eine seltene Erscheinung. In der Regel verwächst das Crus sup. anthelicis mit dem Querschenkel des Helix. Partiell verwachsen fand ich es bei Verbrechern nur in 3,2 Proc., bei Verbrecherinnen in einem einzigen Falle; hingegen $\mathrm{g} a ̈ n z l i c h$ verwachsen bei Verbrechern 2,3, Verbrecherinnen 2,0 Proc., in viel geringerer Anzahl also als bei Geisteskranken und Idioten, bei welchen diese Deformität zwischen 2-10 Proc. schwankt.

V. Die an der Fossa scaphoidea gefundenen Anomalien sind ebenfalls sehr interessant. Von anatomischew Standpunkte nennen wir dieselbe dann normal, wenn sie bis zum Läppehen herabreicht und hier plötzlich aufhört; ist nun die Fossa, gleichviel ob länger oder kürzer, ist sie anomal; ebenso wenn sie überbriuckt ist.

Beiderseitig auf das Läppchen sich erstreckende Fossa scaphoidea fand ich bei normalen Individuen 7,6, Geisteskranken 11,5 , Idioten 10,3 und Verbrechern 10,2 Proc,; unter Weibern bei Normalen 8,8, Idioten 9,0, Geisteskranken 8,8 und Verbrecherinnen 9,6 Proc. Beiderseitig verkürzte oder mangelhaft entwickelte Fossa scaphoidea fand ich bei Verbrechern 10,5 Proc., Verbrecherinnen 15,2 Proc.; bei Geisteskranken und Idioten ist diese Zahl viel geringer, trotzdem dem Zustandekommen dieser Anomalie die abnorme Gestaltung des Helix und Anthelix Vorschub leistet.

Wenn der Helix mit dem Anthelix vollständig oder partiell convergirt, so nennen wir die Fossa scaphoidea überbrü ckt. Nach meinen Beobachtungen finden wir bei Geisteskranken und Idioten diese Anomalie mit 10-15 Proc. vertreten, also doppelt so viel wie bei Normalen; hingegen fand ich bei Verbrechern 9,5, bei Verbrecherinnen nur 8,9 Poc.

VI. Beim Tragus und Antitragus interessiren blos die Dimensionsanomalien. Mancher Autor zog zwar auch die Gestalt und Lagerung in Betracht, doch lässt sich denselben keine grosse Bedeutung beimessen. Zweifellos ist, dass, während bei normalen 
Individuen diese zwei Erhabenheiten kaum in 1 Proc. der Fälle vergrössert zu finden sind, sie bei Verbrechern mit 5,8 und die beiden zusammen mit 2,1 Proc. vertreten sind; bei Verbrecherinnen fand ich den Tragus in 2,7, den Antitragus in 7,1, beide zugleich in 1,2 Proc. der Fälle vergrössert.

Resumiren wir nun alle diese Daten, so glauben wir bestimmt constatiren zu können, dass bei Geisteskranken, Idioten und Verbrechern die morphologischen Veränderungen der Ohrmuschel viel häufigervorkommen, dass auch von diesen Anomalien insbesondere die bedeutenderen zahlreichvertreten sind, undendlich, dass wir diese Anomalien unter den Geisteskranken, Idioten und Verbrechern bei Männern in viel grösserer Anzabl finden, als bei den ebenso classificirten Weibern.

Schliesslich stellte ich noch Berechnungen dariiber an, in welcher Anzabl die zweiseitigen oder einseitigen Anomalien vorkommen. Von sämmtlichen Anomalien bei

\section{Männern}

Normale Geisteskranke Idioten Verbrecher

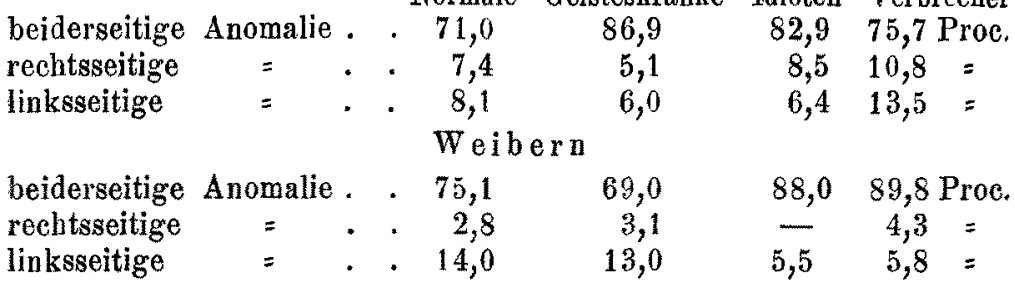

Die Anomalien sind daher überwiegend bilaterale; wenn einseitig, so finden sie sich häufiger linksseitig.

In welcher Anzahl sich die Anomalien an je einem Untersuchten finden, zeigt folgende Tabelle:

Ich fand bei Männern auf je 1 normales Individuum 1,8, Geisteskranken 1,5, Idioten 2,4, Verbrecher 1,4 Proc. Anomalien; bei Weibern auf je 1 Normale 0,5, Geisteskranke 2,1, Idiotin 2,2, Verbrecherin 1,2 Proc. Anomalien, welche Ziffern wieder beweisen, dass bei Geisteskranken, Idioten und Verbrechern a uch auf ein und dasselbe Individum durchschnittlich mehr Anomalien fallen, als auf das normale Individuum. 